\title{
КОНТРАСТНО-УСИЛЕННОЕ УЛЬТРАЗВУКОВОЕ ИССЛЕДОВАНИЕ В ДИФФЕРЕНЦИАЛЬНОЙ ДИАГНОСТИКЕ РАКА ЩИТОВИДНОЙ ЖЕЛЕЗЫ
}

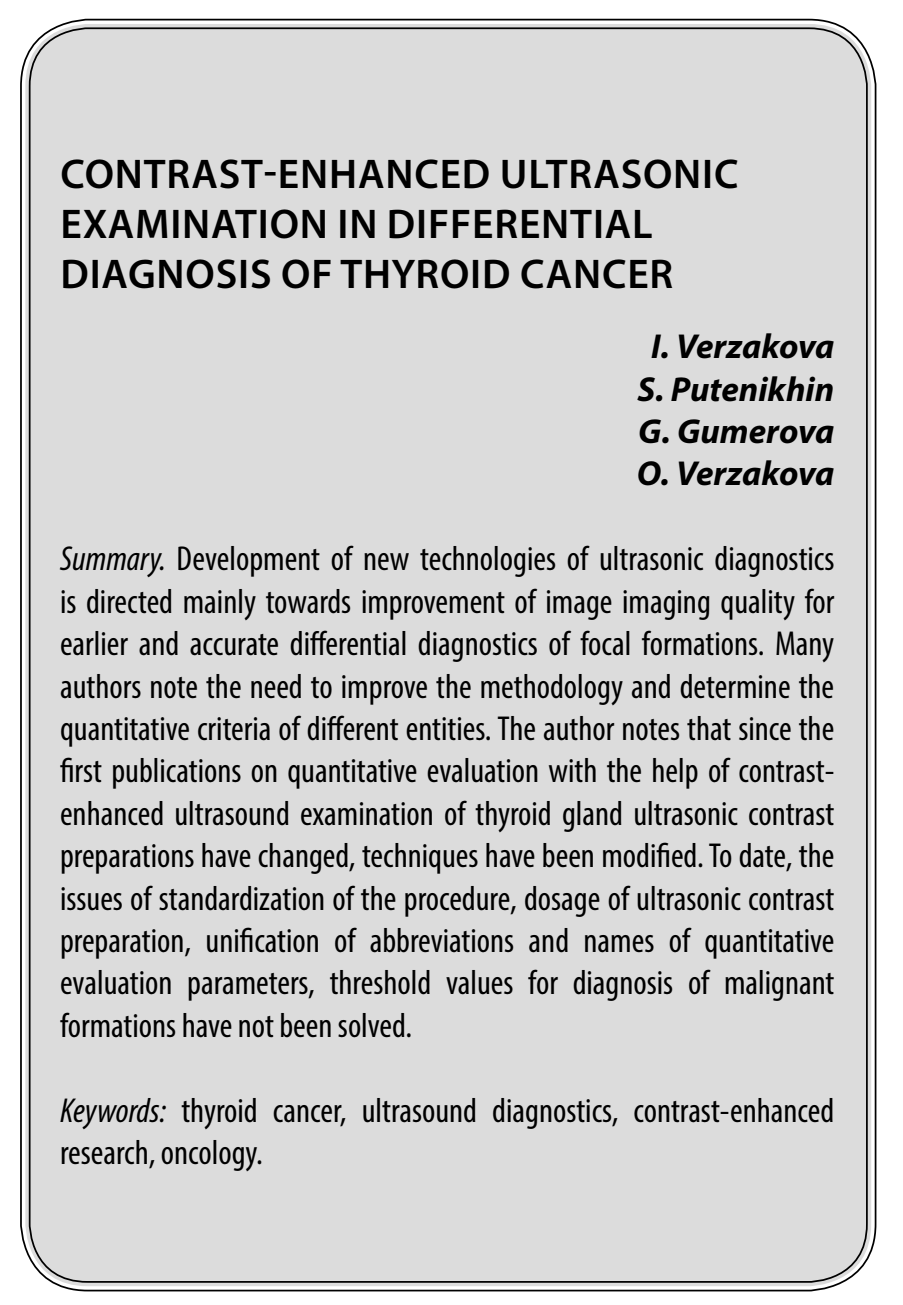

\section{Введение}

$\mathbf{P}$ азвитие новых технологий ультразвуковой диагностики направлено преимущественно в сторону повышения качества визуализации изображения для более ранней и точной дифференциальной диагностики очаговых образований $[8,9]$. В настоящее время имеется большое количество преимущественно зарубежных научных публикаций, посвященных ультразвуковому исследованию щитовидной железы с контрастным усилением (КУУЗИ) и указывающих на перспективность применения ультразвуковых контрастных препаратов в дифференциальной диагностике очаговых образований щитовидной железы [12]. Однако европейские и азиатские исследователи разноречивы по группам и методам анализа, особенно-

\author{
Верзакова Ирина Викторовна \\ Д.м.н., Башкирский государственный медичинский \\ университет, г. Уфра \\ Путенихин Сергей Юрьевич \\ Аспирант, Башкирский государственный \\ медицинский университет, г. Уфа \\ putenihin1970@yandex.ru \\ Гумерова Гульнара Тагировна \\ К.м.н., дочент, Башкирский государственный \\ медицинский университет, г. Уфра \\ Верзакова Ольга Владимировна \\ К.м.н., дочент, Башкирский государственный \\ медицинский университет, г. Уфа
}

Аннотация. Развитие новых технологий ультразвуковой диагностики направлено преимущественно в сторону повышения качества визуализации изображения для более ранней и точной дифференциальной диагностики очаговых образований. Многими авторами отмечается необходимость совершенствования методики, определения количественных критериев различных образований. Автор отмечает, что с момента первых публикаций о проведении количественной оценки с помощью контрастно-усиленного ультразвукового исследования щитовидной железы поменялись ультразвуковые контрастные препараты, модифицировались методики. До настоящего времени вопросы стандартизации проведения процедуры, дозировки ультразвукового контрастного препарата, унификации аббревиатур и наименований параметров количественной оценки, пороговых значений для диагностики злокачественных образований так и не были решены.

Ключевые слова: рак щитовидной железы, УзИ-диагностика, контрастно-усиленное исследование, онкология.

стям научного поиска и результатам КУУЗИ. В большинстве работ оцениваются только качественные характеристики контрастирования [18]. Многими авторами отмечается необходимость совершенствования методики, определения количественных критериев различных образований [8]. В последних рекомендациях по внепеченочному применению ультразвуковых контрастных препаратов отмечено, что в настоящее время КУУЗИ находится на этапе активного научного исследования и не может быть рекомендовано для рутинной клинической практики [9].

\section{Материал и методы}

В основу работы положены результаты ретроспективного сопоставления данных ультразвуковых заклю- 
чений с результатами цитологического и гистологического исследований, проведенных в период с декабря 2017 по сентябрь 2019 г. КУУЗИ щитовидной железы проведено 154 пациентам с узловыми образованиями щитовидной железы в возрасте от 21 до 76 лет. Среди пациентов было 132 (85,7\%) женщины, 22 (14,3\%) мужчины. Гендерное соотношение составляло 6: 1.

Все 73 пациента с раком щитовидной железы прооперированы в хирургическом стационаре, диагноз подтвержден гистологически. Среди них папиллярный рак выявлен у 64 (87,7\%), фолликулярный у 9 (12,3\%) пациентов.

74 пациента с доброкачественными образованиями щитовидной железы также получили хирургическое лечение в хирургическом стационаре, диагноз подтвержден гистологически. 7 пациентов с доброкачественной патологией щитовидной железы получили амбулаторное лечение, диагноз верифицирован по данным тонкоигольной аспирационной пункционной биопсии и цитологического исследования. Среди 81 пациента с доброкачественной патологией щитовидной железы фолликулярная аденома выявлена у 56 (69,1\%), коллоидные узлы у 12 (14,8\%), аутоиммунный тирео идит с узлообразованием у 13 (16,0\%) пациентов.

КУУЗИ проводили на сканере DC8 (Mindly, Китай) с использованием линейного мультичастотного датчика, работающего в диапазоне частот 7,515,0 МГц, в специализированном режиме с низким механическим индексом $(<0,10)$. В качестве ультразвукового контрастного препарата был применен Соновью (Bracco Swiss S.A., Швейцария) в дозировке 2,4 мл.

Предварительно в каждом случае было получено письменное согласие пациента на проведение процедуры ультразвукового контрастирования. Форма информированного согласия и протокол клинического исследования были утверждены согласно нормативным требованиям лечебного учреждения.

Всем 154 пациентам было проведено предварительное мультипараметрическое ультразвуковое исследование щитовидной железы с применением серошкальной эхографии (В режим), цветокодированной допплерографии, импульсно-волновой допплерографии, компрессионной эластографии. Оценивались количество и расположение очагов. В случаях, когда у пациента определялось более одного образования в щитовидной железе, для проведения КУУЗИ выбиралось наиболее удобное для сканирования образование или образование, планирующееся к пункционной биопсии. Определялась оптимальная плоскость сканирования очага при его хорошей визуализации и отсутствии артефактов.
Процедура КУУЗИ проводилась в условиях чистой перевязочной при соблюдении единых требований асептики для проведения малоинвазивных манипуляций. Ультразвуковой контрастный препарат вводился внутривенно болюсно через периферический венозный катетер $20 \mathrm{G}$ в кубитальную вену с последующим введением 5 мл 0,9\%го раствора $\mathrm{NaCl}$. Одновременно с введением Соновью начинались отсчет времени и запись кинопетли до полного вымывания Соновью из образования (не менее 130 с). Проводилась обязательная запись видеопетли (архивирование).

На этапе постобработки выполнялся количественный анализ с помощью программного обеспечения ультразвукового сканера ContrastQA. K анализу были приняты сегменты кинопетли от момента введения Соновью в венозное русло до момента его полного вымывания (не менее 130 с) (объект интереса находился в зоне сканирования все время проведения исследования). Результат оценки был представлен кривой «времяинтенсивность», параметры кривой автоматически отображались на экране прибора в виде табличных данных.

Анализ проводился по следующим количественным параметрам:

1. время до пика интенсивности (TPI (time to peak intensity)) (с) время, за которое интенсивность контрастирования ROCтигает максимума;

2. пик интенсивности (PI (peak intensity)) (дБ) максимум интенсивности контрастирования;

3. время полувыведения (DT/2 (descending time/2)) (c) время, за которое интенсивность контрастирования снижается до половины максимума;

4. скорость снижения контрастирования за 30 с после пика интенсивности (DV (descending velocity)) (дБ/с).

Количественные параметры КУУЗИ на основе анализа кривой «время-интенсивность» были получены для узловых образований и прилегающей паренхимы щитовидной железы без очаговых изменений. По ним были рассчитаны соответствующие индексы и разница.

Статистическую обработку результатов исследования проводили при использовании программ Statistica (версия 12.5) и MedCalc (версия 15.8) в среде Windows.

\section{Результаты}

Медиана среднего размера злокачественных образований составила 16,0 мм, 25-75й процентили 11,0-28,5 мм; доброкачественных образований 27,0 и 16,0-35,0 мм соответственно. Количественные параметры КУУЗИ в группах злокачественных $(n=73)$ и доброкачественных $(n=$ 81) продемонстрировали статистически значимые различия между обеими группами были выявлены по следую- 
щим параметрам: Р1узел, Р1паренхима, DT/2узел, индекс DT/2, DVузел, индекс DV, разница DV $(P<0,05)$.

Однако при проведении ROC анализа не все указанные количественные параметры дают высокие показатели информативности. Для диагностики злокачественных образований щитовидной железы наиболее информативными оказались: индекс DT/2, индекс DV, разница DV.

Тест «индекс DT/2 > 1,028 рак щитовидной железы» характеризуется чувствительностью $86,1 \%$, специфичностью 85,2\%, предсказательной ценностью положительного теста $87,7 \%$, предсказательной ценностью отрицательного теста 83,4\%, площадью под кривой 0,872.

Тест «индекс DV < 0,895 рак щитовидной железы» характеризуется чувствительностью 66,7\%, специфичностью 95,1\%, предсказательной ценностью положительного теста 94,3\%, предсказательной ценностью отрицательного теста 70,0\%, площадью под кривой 0,840.

Тест «разница DV < 0,020 дБ/с рак щитовидной железы» характеризуется чувствительностью 66,7\%, специфичностью 95,1\%, предсказательной ценностью положительного теста 94,3\%, предсказательной ценностью отрицательного теста 70,0\%, площадью под кривой 0,842 .

Количественные параметры КУУЗИ в подгруппах папиллярного $(\mathrm{n}=64)$ и фолликулярного $(\mathrm{n}=9)$ рака щитовидной железы продемонстрировали статистически значимые различия между обеими подгруппами были выявлены по следующим параметрам: индекс TPI, P1yзел, индекс DV $(\mathrm{P}<0,05)$.

При проведении ROC-анализа ни один из указанных выше количественных параметров не дает адекватные показатели информативности при дифференциальной диагностике папиллярного и фолликулярного рака щитовидной железы. Однако необходимо отметить малочисленность подгруппы фолликулярного рака щитовидной железы, что является ограничением нашего исследования и требует продолжения исследования в этом направлении.

Количественные параметры КУУЗИ в подгруппах фолликулярной аденомы ( $\mathrm{n}=56)$, коллоидных узлов ( $\mathrm{n}=12$ ) и аутоиммунного тиреоидита с узлообразованием $(n=13)$ показали статистически значимые различия были выявлены по следующим параметрам: ТР1узел, TP1паренхима, индекс ТР1, Р1узел, Р1паренхима, индекс $\mathrm{P} 1$, индекс DT/2 $(\mathrm{P}<0,05)$.

Однако при проведении ROC-анализа ни один из указанных выше количественных параметров не дает адек- ватные показатели информативности при дифференциальной диагностике различных доброкачественных образований. Также ограничивает анализ малочисленность подгрупп коллоидных узлов и аутоиммунного тиреоидита с узлообразованием.

\section{Обсужление}

Опухолевый ангиогенез напрямую зависит от вазогенного эндотелиального фактора роста опухоли, который способствует формированию аномальной сосудистой сети, питающей растущую опухоль. КУУЗИ позволяет визуализировать дезорганизо ванность сосудистого рисунка, штопороподобный ход интранодулярных и перинодулярных сосудов, разрозненность их пространственного расположения [8].

На сегодняшний день КУУЗИ щитовидной железы оценивается лишь как дополнительный метод диагностики злокачественных и доброкачественных узловых образований, предшествующий гистологическому исследованию [12]. Однако уже накоплен ROC-достаточный опыт в оценке эффективности КУУЗИ в дифференциальной диагностике узловых образований щитовидной железы, чтобы утверждать, что применение ультразвуковых контрастных препаратов повышает диагностическую точность рутинного ультразвукового исследования и может помочь улучшить определение зон и узлов для проведения процедуры тонкоигольной аспирационной пункционной биопсии [14].

До появления соответствующего технического программного обеспечения ультразвуковых аппаратов КУУЗИ щитовидной железы включало лишь визуальную оценку качественных характеристик контрастирования. Безусловно, количественный анализ затрудняет процедуру КУУзИ, однако введение количественной оценки снижает риск субъективизма и обеспечивает более объективные и воспроизводимые данные [10].

С момента первых публикаций о проведении количественной оценки КУУЗИ щитовидной железы поменялись ультразвуковые контрастные препараты, модифицировались методики. До настоящего времени вопросы стандартизации проведения процедуры, дозировки ультразвукового контрастного препарата, унификации аббревиатур и наименований параметров количественной оценки, пороговых значений для диагностики злокачественных образований так и не были решены. Приемлемые показатели информативности параметров количественного анализа КУУЗИ щитовидной железы позволяют использовать их в дифференциальной диагностике злокачественных и доброкачественных узловых образований. Однако с целью решения обозначенных выше вопросов необ- 
ходимо проведение дальнейших исследований на более многочисленных группах пациентов различных нозологий.

\section{Зак^ючение}

Таким образом, в нашем исследовании мы показали, что проведение контрастно-усиленного ультразвукового исследования в дифференциальной диагностике рака щитовидной железы представляет актуальное направление для проведения научных изысканий на сегодняшний день. И хотя нами были не выявлены диагностически значимые методики проведения исследования для дифференцировки отдельных нозологических форм, тем не менее показана высокая чувствительность и специфичность исследуемой методики в силу обладания последней высокой специфичности и чувствительности, в рассматриваемых нами тестах.

\section{ЛИТЕРАТУРА}

1. Практическое руководство по ультразвуковой диагностике. Общая ультразвуковая диагностика. Изд. 3-е / Под ред. В. В. Митькова. М.: Видар, 2019. 720 с.

2. Бельцевич Д.Г., Ванушко В. Э. Современные аспекты диагностики узлового зоба // Эндокринная хирургия. 2018. № 3. С. 5-13.

3. Зубарев А.В., Федорова А. А., Чернышев В.В., Варламов Г. В., Соколова Н. А., Федорова Н. А. Применение эхоконтрастных препаратов в клинике и перспективы синхронизации УЗИ, КТ- и МРТ-изображений (собственный опыт и обзор литературы) // Медицинская визуализация. 2015. № 1.С.94-114.

4. Каприн А.Д., Старинский В. В., Петрова Г. В. Состояние онкологической помощи населению России в 2016 году. М.: МНИОИ им. П. А. Герцена - филиал ФГБУ «НМИРЦ» Минздрава России, 2017. 236 с.

5. Смирнова В.А., Семкина Г. В., Платонова Н. М., Ванушко В. Э. Папиллярная микрокарцинома щитовидной железы // Клиническая и экспери-ментальная тиреоидология. 2015. Т. 11. № 2. C.11-24.

6. Cantisani V., Consorti F., Guerrisi A., Guerrisi I., Ricci P., Di Segni M., Mancuso E., Scardella L., Milazzo F., D'Ambrosio F., Antonaci A. Prospective comparative evaluation of quantitative-elasto- sonography (Q-elastography) and contrast- enhanced ultrasound for the evaluation of thyroid nodules: preliminary experience // Eur. J. Radiol. 2013. V. 82. No. 11. P. 1892-1898.

7. Cooper D.S., Doherty G.M., Haugen B. R., Kloos R. T., Lee S. L., Mandel S. J., Mazzaferri E. L., Mclver B., Sherman S. I., Tuttle R. M.; American Thyroid Association Guidelines Taskforce. Management guidelines for patients with thyroid nodules and differentiated thyroid cancer // Thyroid. 2016. V. 16. No. 2. P. 109-142.

8. Fleischer A.C., Niermann K. J., Donnelly E. F., Yankeelov T. E., Canniff K. M., Hallahan D. E., Rothenberg M. E. Sonographic depiction of microvessel perfusion: principles and potential // J. Ultrasound Med. 2015. V. 23. No. 11. P. 14991506.

9. Giusti M., Campomenosi C., Gay S., Massa B., Silvestri E., Monti E., Turtulici G. The use of semi- quantitative ultrasound elastosonography in combination with conventional ultrasonography and contrast-enhanced ultrasonography in the assessment of malignancy risk of thyroid nodules with indeterminate cytology // Thyroid Res. 2017. V. 7. No. 1. P. 9.

10. Hu Y., Li P., Jiang S., Li F. Quantitative analysis of suspicious thyroid nodules by contrast-enhanced ultrasonography // Int. J. Clin. Exp. Med. 2018. V. 8. No. 7. P. 11786-11793.

11. Jiang J., Huang L., Zhang H., Ma W., Shang X., Zhou Q., Gao Y., Yu S., Qi Y. Contrast-enhanced sonography of thyroid nodules // J. Clin. Ultrasound. 2015. V. 43. No. 3. P. 153-156.

12. La Vecchia C., Malvezzi M., Bosetti C., Garavello W., Bertuccio P., Levi F., Negri E. Thyroid cancer mortality and incidence: a global overview // Int. J. Cancer. 2015. V. 136. No. 9. P. 2187-2195. Doi: 10.1002/ijc.29251.

13. Sun B., Lang L., Zhu X., Jiang F., Hong Y., He L. Accuracy of contrast-enhanced ultrasound in the identification of thyroid nodules: a metaanalysis // Int. J. Clin. Exp. Med. 2015. V. 8. No. 8. P. 12882-12889.

14. Wendl C.M., Janker M., Jung W., Stroszczysn- ski C., Jung E. M. Contrast-enhanced ultrasound with perfusion analysis for the identification of malignant and benign tumours of the thyroid gland // Clin. Hemorheol. Microcirc. 2015. V. 63. No. 2. P. 113-121. Doi: 10.3233/CH-151966.

15. World Health Organization Classification of Tumours. Pathology and Genetics. Tumours of Endocrine Organs / Ed. by DeLillis R., Lloud R., Heitz P. U., Eng C. Lyon: I ARC Press, 2014. P. 49-135.

16. Yu D., Han Y., Chen T. Contrast-enhanced ultrasound for differentiation of benign and malignant thyroid lesions: meta-analysis // 0tolaryngol. Head Neck Surg. 2014. V. 151. No. 6. P. 909-915. Doi: 10.1177/0194599814555838.

17. Zhang Y., Zhou P., Tian S. M., Zhao Y. F., Li J. L., Li L. Usefulness of combined use of contrast- enhanced ultrasound and TI-RADS classification for the differentiation of benign from malignant lesions of thyroid nodules // Eur. Radiol. 2017. V. 27. No. 4. P. 1527-1536.

18. Zhao R.N., Zhang B., Yang X., Jiang Y.X., Lai X.J., Zhu S.L., Zhang X.Y. Diagnostic value of contrast-enhanced ultrasound of thyroid nodules coexisting with Hashimoto's thyroiditis // Zhongguo Yi Xue Ke Xue Yuan Xue Bao. 2015. V. 37. No. 1. P. 66-70. 